\title{
Survival in HIV-infected patients with lymphoma according to the choice of antiretroviral treatment: an observational multicentre study
}

\author{
E Focà iD,${ }^{1, \dagger}$ G Cavaglià, ${ }^{2, \dagger} \mathrm{S}$ Rusconi, ${ }^{3}$ A Cascavilla, ${ }^{4}$ G Cenderello, ${ }^{5}$ A Re, ${ }^{6}$ S Casari, ${ }^{7}$ L van den Bogaart, ${ }^{3}$ PL Zinzani, ${ }^{4}$ \\ D Caracciolo, ${ }^{8}$ G Di Perri, ${ }^{2}$ A Bonito, ${ }^{2}$ A Lucchini, ${ }^{2}$ G Cassola, ${ }^{5}$ P Viale $^{4}$ and A Calcagno ${ }^{2}$ \\ ${ }^{1}$ Department of Infectious and Tropical Diseases, University of Brescia and Brescia Spedali Civili Hospital, Brescia, Italy, \\ ${ }^{2}$ Unit of Infectious Diseases, Department of Medical Sciences, University of Torino, Torino, Italy, ${ }^{3}$ Department of \\ Infectious Diseases, Ospedale Luigi Sacco, University of Milan, Milan, Italy, ${ }^{4}$ Department of Internal Medicine, Geriatrics \\ and Nephrologic Diseases, Section of Infectious Diseases, S. Orsola-Malpighi Hospital, “Alma Mater Studiorum” \\ University of Bologna, Bologna, Italy, ${ }^{5}$ Department of Infectious Diseases, EO Ospedali Galliera, Genova, Italy, ${ }^{6}$ Unit of \\ Haematology, Spedali Civili, Brescia, Italy, ${ }^{7}$ Unit of Infectious Diseases, Carlo Poma Hospital, Mantova, Italy and ${ }^{8}$ Unit \\ of Haematology, Department of Oncology, University of Torino, Torino, Italy
}

Objectives

Lymphoproliferative disorders are often observed in HIV-positive patients. Combination antiretroviral treatment (cART) during antineoplastic chemotherapy is beneficial, but little is known about the clinical outcome according to different antiretroviral combinations. The aim of the study was to address this gap in current knowledge.

\section{Methods}

A retrospective study was conducted in five large Italian centres for the period from 1998 to 2015; HIV-positive patients diagnosed with lymphoma were included and demographic, clinical and therapeutic variables were recorded and associated with clinical outcomes. Bivariate and multivariate analyses were performed, including Cox proportional hazard models for survival.

Results

A total of 399 patients were included in the study. The most common types of lymphoma were diffuse large B-cell lymphoma (DLCLB; $n=164$ ), Hodgkin lymphoma (HL; $n=99$ ) and Burkitt lymphoma (BL; $n=57$ ), followed by plasmablastic lymphoma (PBL; $n=38$ ), T-cell lymphoma (TCL; $n=17)$, indolent lymphoma $(n=10)$ and other less common types $(n=14)$. cART was given to 327 (out of 387 evaluable) patients: in 216 subjects it was protease inhibitor (PI)-based, in 73 it was nonnucleoside reverse transcriptase inhibitor (NNRTI)-based and in 18 it was integrase strand transfer inhibitor (INSTI)-based (the remaining 20 individuals received other regimens). The 5-year overall survival was 57.5\% (52.8\% for DLCLB, 67.8\% for HL, 42.3\% for BL, 60.6\% for PBL and $64.7 \%$ for TCL). PI-based ART compared with other compounds was associated with worse survival in non-Hodgkin lymphoma (NHL) and HL patients combined $(P \leq 0.001)$ and in NHL patients alone $(P<0.001)$; grade 3-4 haematological toxicities were more commonly observed in PI-treated individuals. Lymphoma diagnosis in recent years, better immunovirological status, lower lymphoma stage and better prognostic indexes were associated with better survival.

\section{Conclusions}

PI-based cART while on chemotherapy was associated with worse overall survival and more frequent haematological complications in HIV-positive patients with lymphoma.

Keywords: drug-to-drug interactions, HIV, lymphoma, protease inhibitors, toxicity

Accepted 15 March 2018

\footnotetext{
Correspondence: Emanuele Focà, MD PhD, Clinic of Infectious and Tropical Diseases, Department of Clinical and Experimental Sciences, School of Medicine, University of Brescia, Piazzale Spedali Civili 1, Brescia 25136, Italy. Tel: +390303995677; fax: +390303996084; e-mail: emanuele.foca@unibs.it 


\section{Introduction}

HIV-related lymphomas (HRLs) include a spectrum of different pathological entities, each representing a major complication in the natural history of HIV infection. Their clinical outcome is associated with variables related both to the severity of the neoplastic disease and to the patients' immunovirological status $[1,2]$. Since the introduction of combination antiretroviral therapy (cART), a remarkable change in HRL incidence and prognosis has been observed. On the one hand, epidemiological data indicate a significant decrease in the absolute incidence of the most aggressive non-Hodgkin lymphoma (NHL) histotypes, and a trend for an increase in the incidence of histotypes with better prognosis such as Hodgkin lymphoma (HL) [3]. On the other hand, a positive direct effect of cART has been suggested, with stronger evidence for an improvement of patients' immunovirological status at lymphoma diagnosis, and more controversial data on cART modulation of chemotherapy-induced immune suppression $[4,5]$. Combining cART with chemotherapy has been shown to reduce the incidence of chemotherapyand AIDS-related infectious complications in HRL patients [6]. Moreover, the rate of complete response (CR) to first-line chemotherapy has been shown to be increased by cART in several studies [6-8]; this resulted in an improvement in overall survival (OS) $[9,10]$.

However, there exists a significant potential for pharmacokinetic (PK) interactions between chemotherapy and antitroviral agents, which stems from their shared metabolic pathways involving hepatic cytochromes. Overall, nonnucleoside reverse transcriptase inhibitors (NNRTIs) as a group induce cytochromes essential for metabolism of chemotherapy agents, while protease inhibitors (PIs) tend to inhibit them. Thus, some argue that NNRTIs share the potential to reduce the concentrations, efficacy and adverse effects of chemotherapy agents, while PIs would exert the opposite effect. Nucleoside reverse transcriptase inhibitors (NRTIs) and integrase strand transfer inhibitors (INSTIs) seem to have very few significant drug-to-drug interactions (DDIs). In vivo $\mathrm{PK}$ studies demonstrated a significant effect of PIs on the area under the curve (AUC) for cyclophosphamide in the Cyclophosphamide, Hydroxydaunorubicin (also called doxorubicin or adramycin), Oncovin (vincristine), Prednisone or Orednisolone (CHOP) regimen [11], and for vinblastine in the Adriamycin (hydroxydaunorubicin/ doxorubicin), Bleomycin, Vinblastine, Dacarbazine (ABVD) regimen [12]. For doxorubicin, there is no apparent effect on plasma concentrations, with some authors suggesting an effect on intracellular concentrations [11,13]. In parallel with a beneficial influence of cART on survival and chemotherapy-related infectious complications, some clinical studies on HRLs have shown an increase in other chemotherapy-related complications, including cytopaenias and neurotoxicity, in patients receiving cART [14,15]. Only a few groups have compared outcomes for patients with HRLs on different cART regimens during chemotherapy [16]. While there is evidence for some influence of the cART subtype on various clinical outcomes, the magnitude of the effect has been found to vary in different studies. A prospective study in NHL patients on the cyclophosphamide, doxorubicin, etoposide (CDE) infusional chemotherapy regimen reported a significant association between PI-based cART and a higher incidence of grade 3-4 infections, grade 4 neutropaenia and lower chemotherapy dose-density [17]. A significantly higher incidence of severe cytopaenias was observed in patients on PI-based cART receiving $A B V D$ chemotherapy for HL [18]. A Korea-based study showed no evidence of an association between PI use and the incidence of febrile neutropaenia in HRL patients [19]. A higher CR rate in HRL patients on PI-based cART was suggested in a retrospective series of 34 patients, but there was an unequal distribution of lymphoma-related prognostic variables among the study groups [20].

The potential for PK interactions seems limited for CART regimens based on the unboosted integrase inhibitor (INI) raltegravir, and observational data show their feasibility and efficacy for HRL patients on chemotherapy [21].

The aim of the study was to analyse the choice of antiretroviral regimens as a prognostic factor and predictor of chemotherapy-associated toxicities in HIV-positive patients diagnosed with lymphomas.

\section{Methods}

We retrospectively identified all consecutive patients diagnosed with HIV-related lymphoma at five Italian institutions between 1998 and 2015. Patients were included if a western blot-confirmed diagnosis of HIV infection and a biopsy-confirmed lymphoma diagnosis were both ascertained. The protocol was approved by each institution's review board and all efforts were made to obtain patients' written consent.

Clinical records were retrospectively reviewed for the following clinical characteristics: age, gender, ethnicity, HIV risk group, hepatitis C virus (HCV) and hepatitis B virus (HBV) infection status, comorbidity burden, duration of HIV infection prior to lymphoma diagnosis and AIDS-defining events (remote or concurrent with lymphoma diagnosis). We divided the study time into three different periods: 1998-2003, 2004-2008 and 2009-2015.

Viro-immunological parameters were CD4 T-cell count and HIV RNA level at different time-points for the CD4 count, the historic CD4 count nadir 'baseline' at lymphoma 
diagnosis, the CD4 count 3 months into chemotherapy and that at chemotherapy completion were used.

Patients who received cART were categorized according to whether they were cART experienced or naïve prior to lymphoma diagnosis and according to their baseline cART regimen at the start of chemotherapy.

The characteristics of the lymphoma were recorded as lymphoma histotype, lymphoma staging according to the Ann Arbor system, the number of extranodal sites, the prognostic score (International prognostic index (IPI) and International prognostic score (IPS), respectively, for NHL and HL) and the chemotherapy regimen.

Adverse events were expressed as the presence of significant reductions in dose-density as a result of chemotherapy toxicity, infectious complications, occurrence of haematological toxicity grade 3 or 4 according to the Common Terminology Criteria for Adverse Events (CTCAE) classification, the occurrence of neuropathy, the occurrence of relapse, overall survival, and cause of death.

Prognostic scores were categorized as follows: low (IPI 0-1; IPS 0-1), intermediate (IPI 2-3; IPS 2-4) and high risk (IPI 4-5; IPS 5-7). These categories were analysed according to descriptive statistics.

Comorbidity burden was expressed as an adjusted Charlson comorbidity index, not including AIDS in the calculated score. A significant dose-density reduction was defined as either a delay in chemotherapy completion of more than a third of the scheduled duration, or a $>30 \%$ dose reduction for at least one chemotherapy agent, in both cases in response to chemotherapy-related toxicity. Infectious complications were categorized as typical chemotherapy-associated infections (e.g. febrile neutropaenia, sepsis, pneumonia or urinary tract infection) or HIV-specific opportunistic infections (e.g. Candida oesophagitis, Pneumocystis jirovecii pneumonia or tuberculosis). Neuropathy was defined either by Electromyography (EMG) or by obvious clinical features as evident in clinical records.

For univariate analysis, $\chi^{2}$, Kruskall-Wallis tests and Kaplan-Meier models were used. Factors with $P<0.10$ in univariate analysis were included in a multivariate model for independence of association. Stepwise selection models were applied to binary logistic regression analysis (dichotomic variables of interest) and to Cox proportional hazard models (survival). Binary logistic regression for identifying variables associated with chemotherapyrelated complications was adjusted for baseline CD4 count and cART use (none vs. PI-based vs. other antiretrovirals) and for variables with $P<0.10$ in univariate analysis. All statistical calculations were performed with the SPSS program version 23.0 (IBM SPSS Statistics. Armonk, NY, United States).

\section{Results}

\section{Baseline characteristics}

Three hundred and ninety-nine patients were included in the study. They were mostly male $(323 ; 81.1 \%)$ and their median age was 43.6 years [interquartile range (IQR) 38.2-50.5 years]. The median baseline CD4 T-cell count and CD4/CD8 ratio were 218 (IQR 102-348) cells/ $\mu \mathrm{L}$ and 0.30 (0.15-0.51), respectively. One hundred and twenty subjects were cART-naïve at lymphoma diagnosis [with a median HIV RNA of $4.91 \log _{10}$ HIV-1 RNA copies/mL (IQR 4.38-5.48) at lymphoma diagnosis], while 264 were on cART, with 114 (43\%) showing plasma HIV RNA < 50 copies/mL. AIDS-related comorbidities were observed in 125 patients (32.6\%); 267 individuals (66.9\%) had a high general comorbidity burden (Charlson index $>1$ ).

Patients were diagnosed with diffuse large B-cell lymphoma (DLCLB; 164 patients), HL (99 patients), Burkitt lymphoma (BL; 57 patients), plasmablastic lymphoma (PBL; 38 patients), indolent lymphoma (10 patients), T-cell lymphoma (17 patients) and other less common types (four patients). Among the three most prevalent lymphoma histotypes (DLBCL, HL and BL), there were no statistically significant differences in terms of gender, ethnicity, HIV risk group and year of lymphoma diagnosis (Table 1). At the time of lymphoma diagnosis, patients with HL had a higher CD4 percentage (22\% vs. $13.4 \%$ for DLCLB and 14.8\% for BL; $P<0.001)$ and lower HIV RNA levels (44.4\% suppressed vs. 30\% for DLCLB and 23\% for BL; $P<0.001$ ) compared with other histologies. Overall, most patients had advanced stage lymphoma (stage $3-4,283 ; 70.9 \%$ ) and intermediate/high prognostic score results (IPI/IPS $>2$, $249 ; 79 \%)$. HL patients had a higher incidence of bone marrow involvement $(36.0 \%$ vs. $20.4 \%$ for DLCLB and $27.5 \%$ for BL; $P=0.034$ ) and a lower risk of extranodal involvement other than bone marrow (21.7\% vs. 49.7\% for DLCLB and 61.1\% for BL; $P<0.001)$.

\section{Antiretroviral and antineoplastic treatments}

Among 387 evaluable patients, 327 (84.5\%) received ART after lymphoma diagnosis: PI-based in 216 (55.8\%), NNRTI-based in 73 (18.8\%), INSTI-based in 18 (4.6\%), NRTI-only-based in 12 patients (2.1\%) and other combinations in eight individuals $(2.1 \%)$.

Details of chemotherapy regimens were available in 367 patients; 11 subjects (3.1\%) did not start any antineoplastic agent, while 356 patients (89.2\%) were treated with chemotherapy or other treatment for lymphoma. Among patients with DLCLB, 109 of 152 evaluable patients received $\mathrm{CHOP}$ or $\mathrm{CHOP}-$ like regimens (71.7\%), $12 \mathrm{CDE}$ (7.9\%), 11 M-BACOD (methotrexate with leucovorin, 
Table 1 Patients' characteristics at baseline according to the five main lymphoma histogroups

\begin{tabular}{|c|c|c|c|c|c|}
\hline Variable & DLCLB & $\mathrm{HL}$ & $\mathrm{BL}$ & PBL & TCL \\
\hline$n$ & 164 & 99 & 57 & 38 & 17 \\
\hline Age (years) & $43(38-49)$ & $45(40-53)$ & $42(35-50)$ & $43(38-52)$ & $44(37-55)$ \\
\hline Gender (male) & $127(77)$ & $78(78.8)$ & $50(87.7)$ & $35(92.1)$ & $13(76.5)$ \\
\hline \multicolumn{6}{|l|}{ Geographical origin } \\
\hline Europe & $141(92.2)$ & $91(96.8)$ & $55(98.2)$ & $35(92.1)$ & 15 (93.8) \\
\hline Africa & $8(5.2)$ & $0(0)$ & $1(1.8)$ & $2(5.3)$ & $0(0)$ \\
\hline Other & $2(1.3)$ & $2(2.1)$ & $0(0)$ & $0(0)$ & $0(0)$ \\
\hline \multicolumn{6}{|l|}{ Risk factor for HIV acquisition } \\
\hline IDU & $56(41.8)$ & $32(39.0)$ & $11(19.6)$ & $7(21.9)$ & $7(46.7)$ \\
\hline MSM & $23(15.0)$ & $19(23.2)$ & $13(23.2)$ & $13(40.6)$ & $4(26.7)$ \\
\hline Heterosexual & $55(35.9)$ & $26(31.7)$ & $23(41.1)$ & $12(27.5)$ & $4(26.7)$ \\
\hline \multicolumn{6}{|l|}{ Calendar year } \\
\hline $1998-2003$ & $51(31.3)$ & $28(28.3)$ & $11(19.3)$ & $6(15.8)$ & $10(58.8)$ \\
\hline 2004-2008 & $57(35.0)$ & $28(28.3)$ & $26(45.6)$ & $9(23.7)$ & $5(29.4)$ \\
\hline CD4 T Iymphocytes (cells/ $\mu \mathrm{L}$ ) & $202(91-363)$ & $250(135-348)$ & $208(115-341)$ & $234(87-440)$ & $127(47-306)$ \\
\hline Baseline $\mathrm{CD} 4 / \mathrm{CD} 8$ ratio & $0.26(0.13-0.40)$ & $0.42(0.26-0.70)$ & $0.30(0.12-0.44)$ & $0.30(0.10-0.50)$ & $0.21(0.05-0.37)$ \\
\hline HIV RNA ( $\log _{10}$ copies/mL) & $4.16(1.69-5.18)$ & $1.69(<1.69-4.08)$ & $4.12(1.69-5.15)$ & $2.01(1.69-4.76)$ & $3.66(2.35-4.91)$ \\
\hline Plasma HIV RNA $<50$ copies $/ \mathrm{mL}$ & $39(27.5)$ & $46(53.5)$ & $13(25.5)$ & $15(45.5)$ & $2(12.5)$ \\
\hline Time between HIV and lymphoma diagnoses (years) & $0.7(0.1-15.8)$ & $6.9(2.5-18)$ & $2(1-9.4)$ & $1.1(0-4.5)$ & $10.6(0.6-4.1)$ \\
\hline Carlson comorbidity index $>1$ & $114(69.5)$ & $61(61.6)$ & $36(63.2)$ & $25(65.8)$ & $11(64.7)$ \\
\hline Advanced lymphoma stage (III/IV) & $118(77.1)$ & $64(74.4)$ & $48(84.2)$ & $32(94.1)$ & $9(64.3)$ \\
\hline Bone marrow involvement & $29(20.4)$ & $32(36)$ & $4(27.5)$ & $9(24.3)$ & $3(21.4)$ \\
\hline Extranodal involvement ( $\geq 1$ site apart from bone marrow) & $77(49.7)$ & $18(21.7)$ & $33(61.1)$ & $20(54.1)$ & $7(46.7)$ \\
\hline \multicolumn{6}{|l|}{ Prognostic score } \\
\hline Low risk & $32(29.1)$ & $13(24.1)$ & $11(20)$ & $5(14.7)$ & $2(18.2)$ \\
\hline Intermediate risk & $72(43.9)$ & $26(48.1)$ & $25(45.5)$ & $18(52.9)$ & $7(63.3)$ \\
\hline High risk & $41(28.3)$ & $15(27.8)$ & $19(34.5)$ & $11(32.4)$ & $2(18.2)$ \\
\hline
\end{tabular}

Values are $n(\%)$ or median (interquartile range).

Prognostic scores: Iow (IPI 0-1; IPS 0-1), intermediate (IPI 2-3; IPS 2-4) and high risk (IPI 4-5; IPS 5-7).

BL, Burkitt lymphoma; DLCBL, diffuse large B-cell lymphoma; PBL, plasmablastic lymphoma; TCL, T-cell lymphoma; HL, Hodgkin lymphoma; IDU, injecting drug user; MSM, men who have sex with men.

bleomycin, doxorubicin, cyclophosphamide, vincristine, and dexamethasone) (7.2\%), eight ProMACE-CytaBOM (cyclophosphamide, doxorubicin, etoposide cytozar, bleomycin, vincristine, methotrexate and prednisone) (5.2\%), and 10 other chemotherapy regimens. Twenty-four (out of 28 evaluable patients) with PBL received CHOP or CHOPlike regimens (85.7\%). Among patients with HL, 44 of 60 evaluable patients received ABVD (73.3\%). Thirty of 54 evaluable patients (55.5\%) with BL received intensive regimens (containing high-dose methotrexate), and 24 (44.4\%) received standard dose chemotherapy (mainly CHOP). Patients with T-cell (T) NHL and low-grade NHL received mainly CHOP. Most DLCLB and BL patients (62\% and 70\%, respectively) received rituximab treatment and its use increased in more recent years (59\% in 2009-2015 vs. 60\% in 2004-2008 vs. 33.3\% in 1998-2003; $P<0.001)$.

\section{Survival}

Five-year OS was 57.5\%; median follow-up was 95 months (average 153 months; IQR 14-275 months). It varied according to histotype as follows: $52.8 \%$ (DLCLB),
67.8\% (HL), 42.3\% (BL), 60.6\% (PBL) and 64.7\% (T-cell lymphoma; Fig. 1).

For outcome analysis, patients were grouped into three categories: all subjects, patients with NHL (DLCBL, PBL and BL, including rituximab use as a covariate) and those with HL. Results of univariate (log-rank test) and multivariate analysis (Cox proportional hazard model) are shown in Table 2. Survival according to cART choice is depicted in Figure 1. PI coadministration was significantly associated with a higher mortality compared with other antiretrovirals (NNRTI, INSTI and NRTI-only regimens) in all patients $(45.6 \%$ vs. $20.2 \%$, respectively; $P \leq 0.001)$ and in those with NHL $(51.8 \%$ vs. $23.5 \%$, respectively; $P<0.001)$ but not in those with $\mathrm{HL}(34.6 \%$ vs. $19 \%$, respectively; $P=0.211$ ).

In all patients who received a diagnosis in recent years, higher baseline CD4 count and the use of cART avoiding PIs were independently associated with better survival (Table 2). In patients with NHL, younger age, higher baseline CD4 count and the use of cART avoiding PIs were independently associated with better survival. In patients with HL (as a consequence of the smaller sample size), 

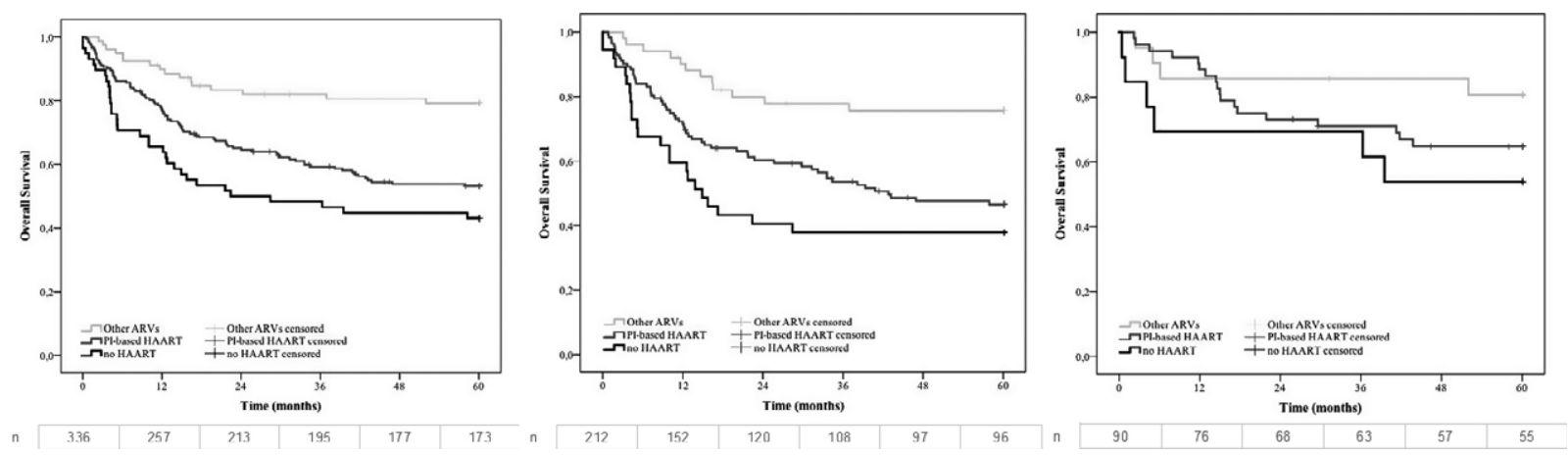

Fig. 1 Survival according to highly active antiretroviral therapy (HAART) use in all patients (left), patients with non-Hodgkin lymphoma (middle) and patients with Hodgkin lymphoma (right). Survival of patients with no HAART started (black lines), those on protease inhibitor (PI)based HAART (grey lines) and those on HAART based on other antiretrovirals (ARVs) (light grey lines) is depicted using Kaplan-Meier curves and compared using log-rank test ( $p<0.001, p<0.001, p=0.211$ for left, middle and right models respectively).

correlated variables were selected according to the strongest association in univariate analysis. In patients with HL, a diagnosis in recent years and bone marrow noninvolvement were associated with better survival.

\section{Dose reductions and toxicity}

The prevalence and predictors of chemotherapy-related complications are shown in Table 3. A PI-containing cART was associated with a higher rate (as compared with other antiretrovirals) of grade 3/4 haematological toxicity in all histotypes. In those receiving antiretrovirals ( $n=323$ ), the prevalence of chemotherapy-associated complications, stratified according to main lymphoma histotypes, is depicted in Figure 2.

\section{Discussion}

In this large retrospective analysis, we have described the determinants of survival in HIV-positive patients presenting with HL and NHL: a diagnosis performed in recent years, better immunovirological status, lower lymphoma stage, better prognostic indexes and the choice of a noninteracting cART were associated with better survival.

Although heterogeneous, this cohort included several patients with various HIV-associated lymphomas presenting features usually associated with severe immune depression such as extranodal and bone marrow involvement, advanced stage and low prognostic indexes [22]. Approximately $70 \%$ of all patients presented comorbidities further complicating the management of the haematological malignancies. HLs were observed in patients who had higher CD4 cell counts and who were more often on ART, as generally reported [23].

Survival was similar to that previously reported in the literature, with approximately 50\% long-term OS in patients with NHL and 65-70\% in patients with HL. Antineoplastic treatments varied by centre and year of diagnosis: the management of HIV-positive subjects improved over time, as demonstrated by the higher survival and the higher use of rituximab in NHL and BL patients in recent years. Conversely, a relatively low number of patients with BL received an infusional intensive antineoplastic regimen (40\%): nevertheless, the 5-year survival was comparable to that of other cohorts, including HIVpositive and HIV-negative subjects [24,25].

The selection of cART had an impact on the survival of the included patients. While those who did not start any treatment were possibly those with worse baseline status for whom treatment was considered inappropriate, PIbased regimens were often used in late-presenting patients. The rationale supporting the use of PIs was the need for high genetic barrier drugs and good immunological recovery in patients presenting with very low CD4 counts. PI-associated DDIs and toxicities started to be reported in 2005 and led to the further suggestion to avoid antiretrovirals that may interact with vinca alkaloids [26-29]. Higher vincristine and vinblastine concentrations have been associated with haematological and neurological toxicities, potentially leading to chemotherapy dose reductions or delays and therefore potentially impairing antineoplastic efficacy. We did not observe a significant effect on these outcomes but we did observe an effect on the incidence of haematological toxicities; in patients with HL, although the effect was nonsignificant, chemotherapy dose reductions and infections were more common in patients on PIs. Additionally, specific factors have been associated with complications during antineoplastic treatment, and they include bone marrow involvement, low CD4 count at baseline and rituximab use. HCV infection was associated, independently of lymphoma histotype, with peripheral neuropathy. Chronic HCV 


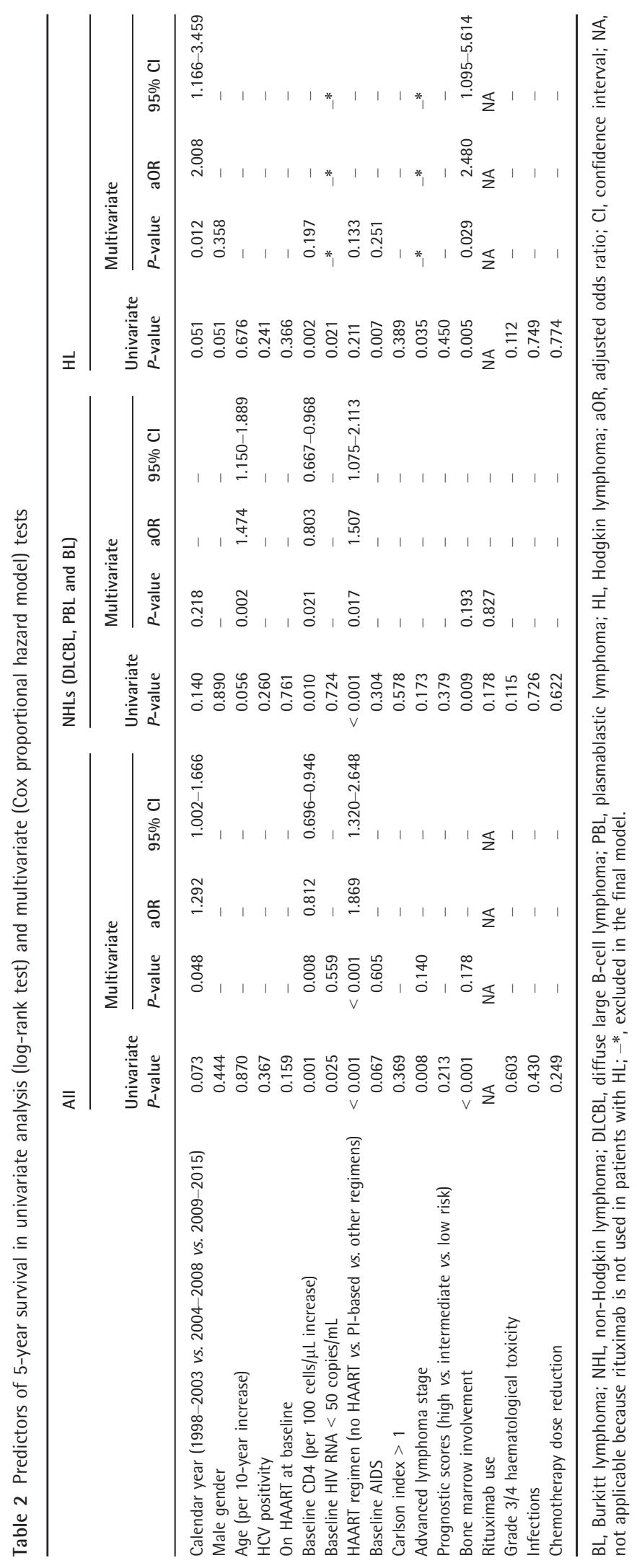


Table 3 Prevalence and predictors of chemotherapy-associated complications

\begin{tabular}{|c|c|c|c|c|}
\hline & & All & NHL & $\mathrm{HL}$ \\
\hline Chemotherapy dose reduction & $\begin{array}{l}n(\%) \\
\text { Predictors in multivariate analysis }\end{array}$ & $\begin{array}{l}55(17.5) \\
\text { None }\end{array}$ & $\begin{array}{l}28 \text { (13.9) } \\
\text { Female gender } \\
\text { Bone marrow involvement } \\
\text { Rituximab use }\end{array}$ & $\begin{array}{l}18(22) \\
\text { None }\end{array}$ \\
\hline Grade $3 / 4$ haematological toxicity & $\begin{array}{l}n(\%) \\
\text { Predictors in multivariate analysis }\end{array}$ & $\begin{array}{l}149 \text { (68.7) } \\
\text { Baseline AIDS } \\
\text { Carlson index }>1 \\
\text { Pl-containing cART }\end{array}$ & $\begin{array}{l}102(67.1) \\
\text { Bone marrow involvement } \\
\text { Carlson index }>1 \\
\text { Pl-containing cART }\end{array}$ & $\begin{array}{l}37 \text { (77.1) } \\
\text { PI-containing cART }\end{array}$ \\
\hline Peripheral neuropathy & $\begin{array}{l}n(\%) \\
\text { Predictors in multivariate analysis }\end{array}$ & $\begin{array}{l}39 \text { (18.5) } \\
\text { HCV infection }\end{array}$ & $\begin{array}{l}21 \text { (13.8) } \\
\text { HCV infection }\end{array}$ & $\begin{array}{l}15 \text { (33.3) } \\
\text { HCV infection }\end{array}$ \\
\hline Infection & $\begin{array}{l}n(\%) \\
\text { Predictors in multivariate analysis }\end{array}$ & $\begin{array}{l}134 \text { (62.3) } \\
\text { Baseline CD4 }\end{array}$ & $\begin{array}{l}100(64.5) \\
\text { Baseline CD4 } \\
\text { Rituximab use }\end{array}$ & $\begin{array}{l}23(53.5) \\
\text { None }\end{array}$ \\
\hline Opportunistic infection & $\begin{array}{l}n(\%) \\
\text { Predictors in multivariate analysis }\end{array}$ & $\begin{array}{l}70(32.7) \\
\text { None }\end{array}$ & $\begin{array}{l}49(31.8) \\
\text { High risk (IPI) } \\
\text { Rituximab use }\end{array}$ & $\begin{array}{l}15(34.9) \\
\text { None }\end{array}$ \\
\hline
\end{tabular}

cART, combination antiretroviral therapy; HCV, hepatitis C virus; NHL, non-Hodgkin lymphoma; HL, Hodgkin lymphoma; PI, protease inhibitor.

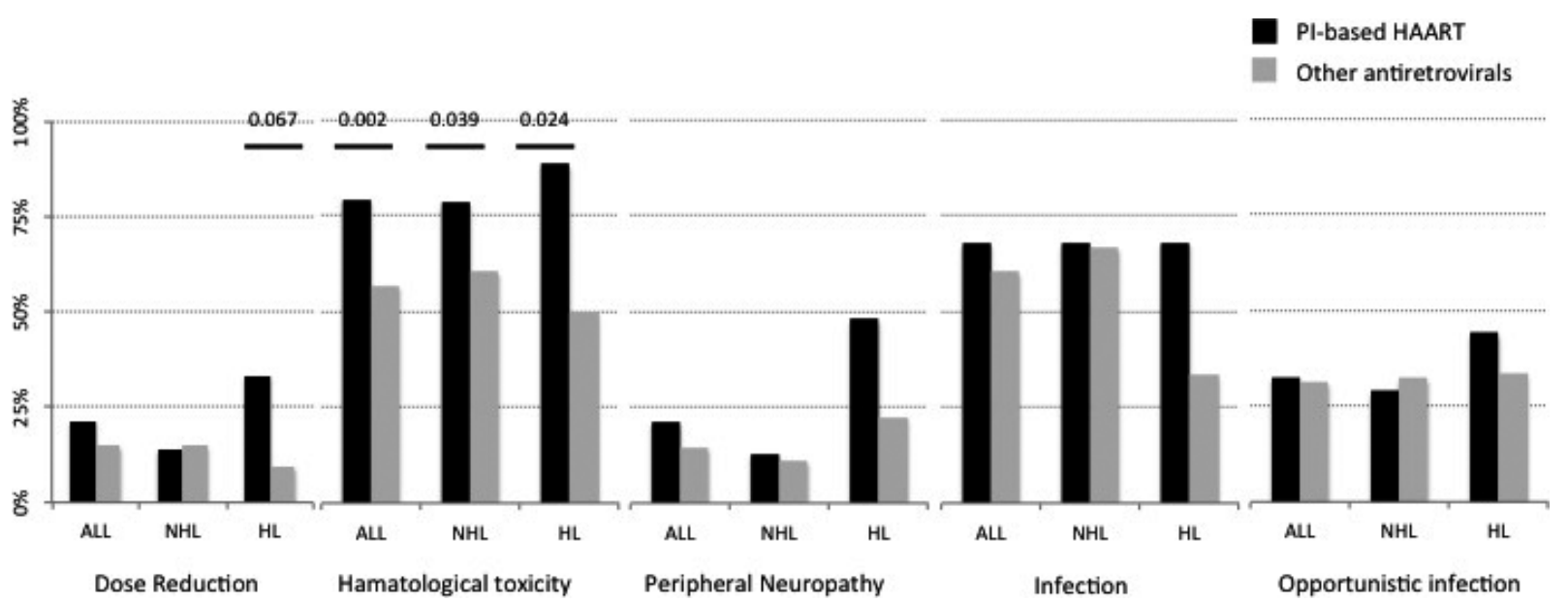

Fig. 2 Prevalence of chemotherapy-associated complications according to main lymphoma histotypes and antiretrovirals [protease inhibitors (PIs) vs. non-protease inhibitors] in highly active antiretroviral therapy (HAART) recipients. Significant univariate associations ( $\chi^{2}$ or Fisher's exact test) are shown above the bars. NHL, non-Hodgkin lymphoma; HL, Hodgkin lymphoma.

infection may cause peripheral neuropathy but it has not been identified as a risk factor during chemotherapy: higher antineoplastic drug concentrations or pre-existing subclinical neuropathy can explain this finding and warrant caution in $\mathrm{HIV} / \mathrm{HCV}$-infected patients presenting with lymphomas.

In the multivariate analysis for survival, different prognostic factors emerged. In NHLs, older age, lower baseline CD4 count and PI use were associated with the risk of death. Prognostic indexes were not retained in the final model, although they were previously validated in other cohorts; the bias of PI administration to patients with a worse clinical status or with late presentation of HIV infection may have affected this finding. In HLs, only earlier year of diagnosis and bone marrow involvement were found to predict mortality: the smaller sample size was probably insufficient to include all factors in the analysis.

Some limitations of this study should be acknowledged, including the large time-span, the heterogeneous management of patients in different clinical sites, the nonstandardized use of antineoplastic regimens, and the incomplete information on causes of death. Additionally, the relatively small sample size did not allow us to analyse the determinants of death in less common histotypes.

Despite earlier findings of PI-associated toxicities during chemotherapy, no study has reported an association between the use of PIs and survival: our results strengthen the recommendation to avoid such compounds and support the use of noninteracting drugs such as integrase inhibitors. The patients included in this study were 
on raltegravir but, given the similar pharmacokinetic pathways, the observed results may reasonably be extended to dolutegravir-containing regimens.

In conclusion, our study demonstrates that, even in recent years, HIV-associated lymphomas have been common in HIV-positive patients and that the choice of a DDI-sparing cART regimen is paramount to enhance survival in this fragile population.

\section{Acknowledgements}

Conflict of interest: EF has received travel grants, consultancy fees and speaker's honoraria from Gilead, ViiV Healthcare, Janssen-Cilag and MSD. SR has been an advisor for Gilead Sciences, AbbVie, BMS, Janssen-Cilag, ViiV Healthcare and MSD, has received speakers' honoraria from Gilead Sciences, ViiV Healthcare, BMS, MSD, AbbVie, and Janssen-Cilag, has received support for travelling to meetings from Gilead Sciences, BMS, JanssenCilag, MSD, and ViiV Healthcare, and has received research grants from Pfizer, Janssen, Gilead Sciences, and ViiV Healthcare. GDP has received grants, travel grants and speaker's honoraria from Abbvie, Gilead, ViiV Healthcare, Janssen-Cilag and MSD. AC has received grants, travel grants and speaker's honoraria from Abbvie, Gilead, ViiV Healthcare, Janssen-Cilag and MSD.

\section{References}

1 Mounier N, Spina M, Gabarre J et al. AIDS-related non Hodgkin lymphoma: final analysis of 485 patients treated with riskadapted intensive chemotherapy. Blood 2006; 107: 3832-3840.

2 Gopal S, Patel MR, Yanik EL et al. Association of early HIV viremia with mortality after HIV-associated lymphoma. AIDS 2013; 27: 2365-2373.

3 Hoffmann C, Hentrich M, Gillor D et al. Hodgkin lymphoma is as common as non-Hodgkin lymphoma in HIV-positive patients with sustained viral suppression and limited immune deficiency: a prospective cohort study. HIV Med 2015; 16: 261-264.

4 Simonelli C, Zanussi S, Cinelli R et al. Impact of concomitant antiblastic chemotherapy and highly active antiretroviral therapy on human immunodeficiency virus (HIV) viremia and genotyping in HIV-infected patients with non-Hodgkin lymphoma. Clin Infect Dis 2003; 37: 820-827.

5 Bower M, Stebbing J, Tuthill M et al. Immunologic recovery in survivors following chemotherapy for AIDS-related nonHodgkin lymphoma. Blood 2008; 111: 3986-3990.

6 Evison J, Jost J, Lederberger B, Jost L, Strasser F, Weber R. HIV-associated non-Hodgkin's lymphoma: highly active antiretroviral therapy improves remission rate of chemotherapy. AIDS 1999; 13: 732-734.
7 Vaccher E, Spina M, Talamini R et al. Improvement of systemic human immunodeficiency virus-related nonHodgkin lymphoma outcome in the era of highly active antiretroviral therapy. Clin Infect Dis 2003; 37: 1556-1564.

8 Hoffmann C, Wolf E, Fatkenheuer G et al. Response to highly active antiretroviral therapy strongly predicts outcome in patients with AIDS-related lymphoma. AIDS 2003; 17: 15211529.

9 Barta SK, Xue X, Wang D et al. Treatment factors affecting outcomes in HIV-associated non-Hodgkin lymphomas: a pooled analysis of 1546 patients. Blood 2013; 122: 3251-3262.

10 Hoffmann C, Chow KU, Wolf E et al. Strong impact of highly active antiretroviral therapy on survival in patients with human immunodeficiency virus-associated Hodgkin's disease. Br J Haematol 2004; 125: 455-462.

11 Ratner L, Lee J, Tang S et al. Chemotherapy for human immunodeficiency virus-associated non-Hodgkin's lymphoma in combination with highly active antiretroviral therapy. $J$ Clin Oncol 2001; 19: 2171-2178.

12 Corona G, Vaccher E, Spina M, Toffoli G. Potential hazard drug-drug interaction between boosted protease inhibitors and vinblastine in HIV patients with Hodgkin's lymphoma. AIDS 2013; 27: 1033-1035.

13 Toffoli G, Corona G, Cattarossi G et al. Effect of highly active antiretroviral therapy (HAARTcART) on pharmacokinetics and pharmacodynamics of doxorubicin in patients with HIV-associated non-Hodgkin's lymphoma. Ann Oncol 2004; 15: 1805-1809.

14 Spina M, Gabarre J, Rossi G et al. Stanford V regimen and concomitant HAART in 59 patients with Hodgkin disease and HIV infection. Blood 2002; 100: 1984-1988.

15 Vaccher E, Spina M, di Gennaro G et al. Concomitant cyclophosphamide, doxorubicin, vincristine, and prednisone chemotherapy plus highly active antiretroviral therapy in patients with human immunodeficiency virus-related, nonHodgkin lymphoma. Cancer 2001; 91: 155-163.

16 Torres HA, Rallapalli V, Saxena A et al. Efficacy and safety of antiretrovirals in HIV-infected patients with cancer. Clin Microbiol Infect 2014; 20: 0672-0679.

17 Bower M, McCall-Peat N, Ryan N et al. Protease inhibitors potentiate chemotherapy-induced neutropenia. Blood 2004; 104: 2943-2946

18 Cingolani A, Torti L, Pinetti C et al. Detrimental clinical interaction between ritonavir-boosted protease inhibitors and vinblastine in HIV-infected patients with Hodgkin's lymphoma. AIDS 2010; 24: 2408-2412.

19 Park J, Kim TM, Hwang JH et al. Risk factors for febrile neutropenia during chemotherapy for HIV-related lymphoma. J Korean Med Sci 2012; 27: 1468-1471.

20 Wong AY, Marcotte S, Laroche M et al. Safety and efficacy of CHOP for treatment of diffuse large B-cell lymphoma with 
different combination antiretroviral therapy regimens:

SCULPT study. Antivir Ther 2013; 18: 699-707.

21 Fulco PP, Hynicka L, Rackley D. Raltegravir-based HAARTCART regimen in a patient with large B-cell lymphoma. Ann Pharmacother 2010; 44: 377-382.

22 Lim ST, Karim R, Nathwani BN, Tulpule A, Espina B, Levine AM. AIDS-related Burkitt's lymphoma versus diffuse large-cell lymphoma in the pre-highly active antiretroviral therapy (HAART) and HAART eras: significant differences in survival with standard chemotherapy. J Clin Oncol 2005; 23: 4430-4438.

23 Biggar RJ, Jaffe ES, Goedert JJ, Chaturvedi A, Pfeiffer R, Engels EA. Hodgkin lymphoma and immunodeficiency in persons with HIV/AIDS. Blood 2006; 108: 3786-3791.

24 Barnes JA, Lacasce AS, Feng Y et al. Evaluation of the addition of rituximab to CODOX-M/IVAC for Burkitt's lymphoma : a retrospective analysis. Ann Oncol 2011; 22: 1859-1864.

25 Wang ES, Straus DJ, Teruya-Feldstein J et al. Intensive chemotherapy with cyclophosphamide, doxorubicin, high-dose methotrexate/ifosfamide, etoposide, and high-dose cytarabine (CODOX-M/IVAC) for human immunodeficiency virusassociated Burkitt lymphoma. Cancer 2003; 98: 1196-1205.

26 Wong A, Tseng A. 2014 HIV Oncology Handbook. Antiretroviral Interactions with Chemotherapy Regimens.

27 Bower M, Palfreeman A, Alfa-Wali M et al. British HIV Association guidelines for HIV-associated malignancies 2014. HIV Med 2014; 15: 1-92.

28 Hentrich M, Hoffmann C, Mosthaf F et al. Therapy of HIVassociated lymphoma-recommendations of the oncology working group of the German Study Group of Physicians in Private Practice Treating HIV-Infected Patients (DAGNÄ), in cooperation with the German AIDS Society (DAIG). Ann Hematol 2014; 93: 913-921.

29 Berretta M, Caraglia M, Martellotta F et al. Drug-drug interactions based on pharmacogenetic profile between highly active antiretroviral therapy and antiblastic chemotherapy in cancer patients with HIV infection. Front Pharmacol 2016; 7: 71. 